\title{
A Compressive Signal Detection Scheme Based on Sparsity
}

\author{
Shaohua Qin ${ }^{1,2, a}$, Dongyan Chen ${ }^{1}$, Xu Huang ${ }^{1}$, \\ and Leilei $\mathrm{Yu}^{1}$ \\ ${ }^{1}$ School of Control Science and Engineering, Shandong University, China \\ ${ }^{2}$ College of Physics and Electronics, Shandong Normal University, China \\ ${ }^{a}$ qsh@sdu.edu.cn
}

\begin{abstract}
Compressed sensing is a revolutionary technology in the research field of signal processing, which can reconstruct the sparse signal using fewer number of compressive measurements compared with conventional reconstruction methods. Compressed sensing can also be utilized to detect the sparse signal. However, the exact reconstruction operation is not necessary when the system aims to detect such sparse signal. Based on compressed sensing, a new compressive signal detection scheme using the sparsity order of the sparse signal is proposed in this paper. Compared with similar detection scheme using the supports of the sparse signal, the newly proposed scheme requires much fewer number of compressive samples. In particular, the proposed scheme does not require the support prior-information of the sparse signal. Simulation results verify the advantages of the proposed scheme and indicate that the new scheme can achieve better detection performance.
\end{abstract}

Keywords: compressed sensing, signal detection, sparsity order

\section{Introduction}

Compressed sensing (CS) is an emerging framework for efficient acquisition and processing of sparse signals, which enables the reconstruction of sparse signals from a small set of sensing measurements, and the number of measurements can be much smaller than the number of Nyquist rate samples [1,2].

Most of the CS literatures focused on rapidly and accurately reconstructing the sparse signal with the smallest set of measurements. But sometimes the signal we acquired only for the purpose of making a detection, such as the spectrum sensing in cognitive radio[3] or event detection in sensor networks $[4,5]$. In these applications, we do not need the complete information of the signal, this means we do not need to reconstruct the whole signal or even we can solve the detection problem from the measurements directly, without any reconstruction. This is called compressive signal processing (CSP), the theoretical analysis about CSP is proposed in [6, 7].

Most of the compressive signal processing methods are to identify the position of nonzero elements in the sparse signal, which demands fewer measurements than reconstruction, this is called support detection $[8,9]$. The support detection can solve the detection and 
classification problem in strong inference and noise scenarios. But when our goal is only to distinguish the presence or absence of the signal, the exact information of positions is not necessary, what we need to know is whether there are nonzero elements. So this problem can be solved through identifying the number of nonzero elements in the sparse signal directly from the received measurements. If the signal is presence, there must be nonzero elements in the measurements, otherwise, the nonzero elements can not be detected. The number of nonzero elements in the sparse signal is called sparsity order in compressed sensing literatures[10].

The sparsity order has been used to reconstruct the signal in [10] and optimize the signal processing in [11]. In [10], the number of compressive measurements needed for reconstruction is decided according to the estimation of the sparsity order. In [11], the sparsity order is used to determine whether the signal is sparse or not, and different signal processing approaches are selected based on the result.

Different from the above applications, in this paper, the sparsity order detection (SOD) scheme is proposed to detect the sparse signal based on its corresponding sparsity order. In this scheme, the number of nonzero elements of the sparse signal is used to indicate the presence or absence of the sparse signal. Compared with the support detection or exact reconstruction, the proposed scheme demands fewer measurements, because the position and amplitude information of nonzero elements are not necessary. On the other hand, the priori information of the support should be known to the support detection scheme. Such information is used to compare with the estimated value obtained from the measurements. Such priori information is hard to be estimated and the proposed scheme does not such information.

The main contributions of this paper are summarized as follows,

1. The sparsity order detection scheme is firstly proposed to detect the sparse signal based on the number of nonzero elements of the sparse signal,

2. The proposed scheme is low computing complexity, because such scheme does not require the exact reconstruction operation and fewer number of measurements are needed,

3. The priori information of non-zero elements, such as position information and amplitude information, are not required in the proposed scheme.

The remainder of this paper is given as follows. In section 2, the related work is described. The Sparsity Order Detection scheme is proposed in section 3. In section 4, we the simulations are given to verify the proposed scheme. Conclusions are offered in section 5 .

\section{Related Work}

\subsection{Compressed Sensing}

The conventional approach to sample signals follows the celebrated Shannon sampling theorem, that is, the sampling rate should be at least twice the maximum frequency presenting in the signal (the so-called Nyquist rate). The theory of compressive sampling also known as compressed sensing (CS) asserts that one can recover sparse signals from far fewer number of samples or measurements than traditional methods. The CS is a concrete 
protocol for sensing and compressing data simultaneously.

Let $\mathbf{x} \in \mathbb{R}^{N}$ be a signal and $\boldsymbol{\Psi} \in \mathbb{R}^{N \times N}$ be a representation basis or dictionary, then the signal can be expressed in this orthonormal basis as

$$
\mathbf{x}=\mathbf{\Psi} \theta
$$

where $\theta \in \mathbb{R}^{N}$ is the coefficient vector. If all but a few of its entries are zero, i.e. $\|\theta\|_{0} \leq K$, then the signal $\mathbf{x} \in \mathbb{R}^{N}$ is a $\mathrm{K}$-sparse signal.

We can get the sensing measurements $\mathbf{y} \in \mathbb{R}^{N}$ from

$$
\mathbf{y}=\mathbf{\Phi} \mathbf{x}
$$

where $\boldsymbol{\Phi} \in \mathbb{R}^{M \times N}(M<N)$ is the sensing matrix. Here $\mathbf{y}$ is undersampled measurements also known as compressive measurements.

If a matrix A satisfies a condition known as the restricted isometry property (RIP) [12]

$$
\left(1-\delta_{K}\right)\|\mathbf{x}\|_{2}^{2} \leq\|\mathbf{A x}\|_{2}^{2} \leq\left(1+\delta_{K}\right)\|\mathbf{x}\|_{2}^{2},
$$

where $\delta_{K}$ is the isometry constant of matrix $\mathbf{A}$ for sparsity $K$, all subsets of $K$ columns taken from $\mathbf{A}$ are in fact nearly orthogonal [2]. Furthermore, the signal $\mathbf{x}$ can be accurately recovered from the compressive measurements $\mathbf{y}$.

Some random matrices such as Gaussian and Bernoulli random matrices follow the restricted isometry property. For Gaussian and Bernoulli random matrices, the lower bound on the minimum number of measurements needed for the sparse signal reconstruction can be written as

$$
M>C K \log (N / K),
$$

where $C$ is a constant determined by the measurements matrix [2].

Most of reconstruction algorithms concentrate on optimization-based methods, such as $l_{1}$-norm minimization which seeks a set of sparse coefficients $\theta$ by solving the convex optimization program [13]

$$
\hat{\theta}=\arg \min \|\theta\|_{1} \text { subject to } \mathbf{y}=\mathbf{\Phi} \mathbf{x}=\boldsymbol{\Phi} \boldsymbol{\Psi} \theta
$$

Greedy reconstruction algorithms build up a signal approximation iteratively by making locally optimal decisions, such as Matching Pursuit (MP) [14],Orthogonal Matching Pursuit (OMP) [15] and Compressive Sampling Matching Pursuit(CoSaMP) [16].

\subsection{System Model}

The binary Hypothesis Test (HT) usually used to distinguish two given hypotheses $\mathbf{H}_{0}$ and $\mathbf{H}_{1}$ can be written as

$$
\begin{aligned}
& \mathbf{H}_{0}: \mathbf{y}=\mathbf{n} \\
& \mathbf{H}_{1}: \mathbf{y}=\mathbf{x}+\mathbf{n},
\end{aligned}
$$

where $\mathbf{x}$ is the target signal, $\mathbf{n} \sim N\left(0, \sigma^{2} \mathbf{I}_{N}\right)$ is an i.i.d. Gaussian noise with variance $\sigma$, and $\mathbf{y}$ is the sampled measurements at the sensor. Note that the AWGN channel is utilized in this communication circumstance and the channel parameters are set to be unitary for simplicity. 
The Probability of Detection $\left(P_{D}\right)$ using energy detection $(\mathrm{ED})$ can be calculated as $[6,7]$

$$
P_{D}(\alpha)=Q\left(Q^{-1}(\alpha)-\frac{\|\mathbf{x}\|_{2}}{\delta}\right),
$$

where the parameter $\alpha$ is the Probability of False Alarm $\left(P_{F}\right)$, and the $Q$ function is expressed as

$$
Q(z)=\int_{z}^{\infty} \exp \left(-\frac{u^{2}}{2}\right) d u .
$$

In the compressive signal processing (CSP) field, we get compressive measurements from Eq. 2, and the hypothesis test shown in Eq. 6 can be rewritten as

$$
\begin{aligned}
& \mathbf{H}_{0}: \mathbf{y}=\mathbf{\Phi} \mathbf{n} \\
& \mathbf{H}_{1}: \mathbf{y}=\boldsymbol{\Phi}(\mathbf{x}+\mathbf{n}),
\end{aligned}
$$

where $\mathbf{\Phi} \in \mathbb{R}^{M \times N}(M<N)$ is the sensing matrix and $\mathbf{x}$ is a K-sparse signal.

The $P_{D}$ from the compressive measurements can be generated as $[6,7]$

$$
P_{D}(\alpha)=Q\left(Q^{-1}(\alpha)-\frac{\|\mathbf{\Phi} \mathbf{x}\|_{2}}{\delta}\right)
$$

Comparing with Eq. 7 and Eq.10, we can know that the performance of CSP depends on the measurement matrix $\mathbf{\Phi}$. Seeing Eq. 3, the detection performance of CSP can achieve approximately that of the conventional detection but using fewer number of measurements $[6,7]$. Therefore, when we only want to detect the target signal, we can solve this problem without constructing the whole signal. This also is the theory basis of the proposed scheme.

\subsection{Support Detection}

A K-sparse signal $\mathbf{x}$ can also be represented as

$$
\mathbf{x}=\sum_{i=1}^{N} \varphi_{i} \theta_{i}
$$

where $\varphi_{i}$ and $\theta_{i}$ are the $i$-th column of the representation basis matrix and corresponding coefficient respectively.

Let $\Omega:=\left\{i, \theta_{i} \neq 0\right\}$ denotes the subset of nonzero coefficients in the above representation, and $\boldsymbol{\Psi}_{\Omega}$ and $\theta_{\Omega}$ denote the corresponding columns and coefficients, respectively.

Therefore, the sparse signal shown in Eq. 11 can be rewritten as

$$
\mathbf{x}=\boldsymbol{\Psi}_{\Omega} \theta_{\Omega} .
$$

The hypothesis test shown in Eq. 9 can be transformed as

$$
\begin{aligned}
& \mathbf{H}_{0}: \mathbf{y}=\mathbf{\Phi} \mathbf{n} \\
& \mathbf{H}_{1}: \mathbf{y}=\boldsymbol{\Phi}\left(\boldsymbol{\Psi}_{\Omega} \theta_{\Omega}+\mathbf{n}\right)=\boldsymbol{\Phi} \boldsymbol{\Psi}_{\Omega} \theta_{\Omega}+\boldsymbol{\Phi} \mathbf{n}
\end{aligned}
$$

The presence or absence of the target signal can be distinguished through identifying the position of nonzero elements in the sparse signal, and without reconstructing the whole signal. Such scheme is named support detection $[8,9,17]$, which tries to find the nonzero 
coefficients from the compressive measurements and determine whether those coefficients belong to the subset $\theta_{\Omega}$. The support detection scheme uses this approach to identify the presence or absence of the sparse signal.

This scheme is used to solve signal detection problems. Because such method only needs to identify the position of each nonzero element in the sparse signal, but not the amplitude of that, it needs fewer numbers of measurements than reconstruction. The number of measurements $M_{s p}$ needed for support detection used OMP algorithm with no noise is [15]

$$
M_{s p}>2 K \log (N-K) .
$$

It is assumed that the knowledge of the support of the target signal is known and the detector should recover the exact support during the detection process [18]. Moreover, the main result of [18] also allows uncertainty in $K$.

However, if the number of measurements is not enough to recover the exact support, or the support prior-information of the sparse signal is not available, we still can construct the detector using the number of the support, which can be considered as the sign of the presence of the signal. Our scheme is mainly based on this motivation.

\section{Sparsity Order Detection}

\subsection{Sparsity Order}

The sparsity order of the sparse signal $\mathbf{x}$ shown in Eq.11 is defined as the $l_{0}$-norm of its coefficient $\theta_{i}$

$$
S_{\text {so }}=\left\|\theta_{i}\right\|_{0} .
$$

Here $S_{s o}$ is used to denote the number of nonzero elements of $\theta_{i}$. The sparsity order of the signal can be estimated from the compressed measurements $\mathbf{y}$ in Eq.2. Such estimation requires fewer number of measurements than the reconstruction of the target signal and the support detection [10].

The sparsity order can be calculated using the following formula given in [10]

$$
\hat{S}_{s o}=\sum_{i=1}^{N}\left(\left|\hat{\theta}_{i}\right|>\gamma\right),
$$

where $\gamma$ is the threshold defined as $\gamma=(\mu+\delta) / 2, \mu$ is the average absolute value of the signal and $\delta$ is the standard deviation of the noise. The larger coefficients of the support can be found using the greedy algorithms, which is similar with the reconstruction algorithm,but is simpler.

The sparsity order estimation only needs to identify the number of nonzero elements in the sparse signal, but not the position or the amplitude of each nonzero element, so this method needs fewer measurements than support detection or reconstruction.

In Figure 1, we compare the estimation performances of the reconstruction, the support detection and the sparsity order detection in terms of correct rate. We use a $K$-sparse signal $\mathbf{x} \in \mathbb{R}^{N}$ as a example, the sensing matrix used is a random matrix $\mathbf{\Phi} \in \mathbb{R}^{M \times N}, M<N$, the entries of the random matrix are independent and identically distributed according to Gaussian probability distributions, in order to discuss easily and without loss of generality, we can use the identity matrix as the representation basis, i.e., $\Psi=\mathbf{I}^{N \times N}$. 


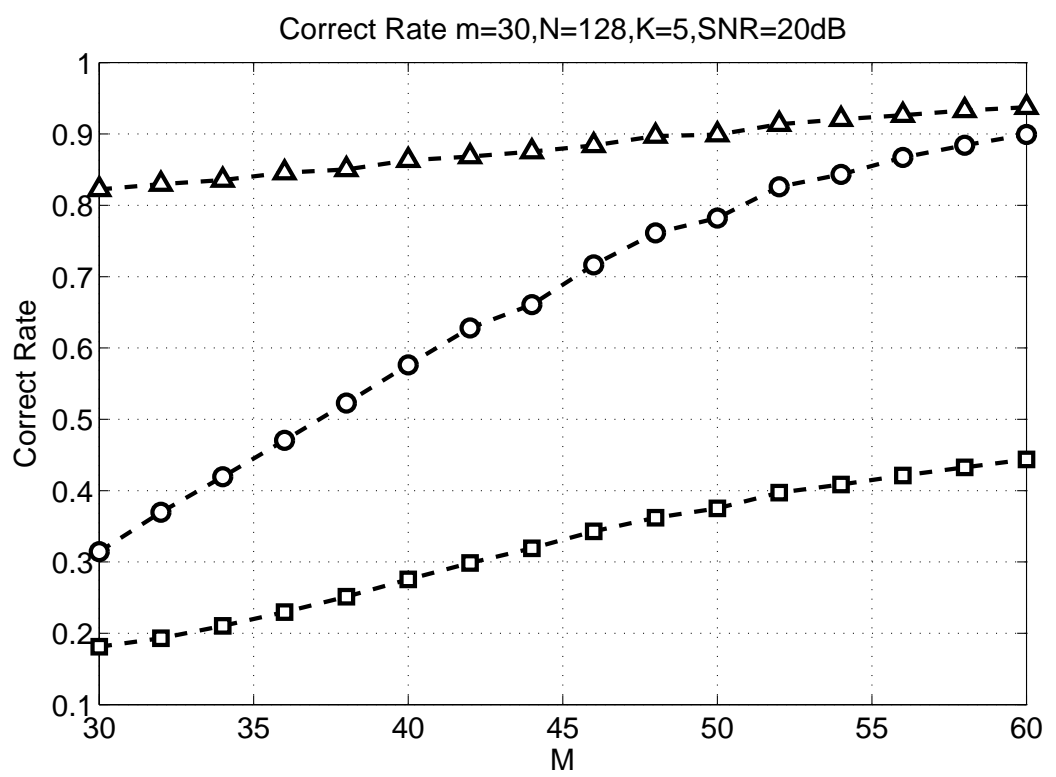

\section{Figure 1. Estimation performance of sparsity order detection and support detection}

The correct rate can be defined as

$$
\begin{aligned}
& C_{R}:=\frac{N_{R E}}{N_{R T}} \\
& C_{S}:=\frac{N_{S E}}{N_{S T}} \\
& C_{O}:=\frac{N_{O E}}{N_{O T}},
\end{aligned}
$$

where $C_{R}, C_{S}$ and $C_{O}$ are the correct rates of the reconstruction,the support estimation and sparsity order estimation, respectively. $N_{R E}$ and $N_{R T}$ are the power of the estimated signal and the power of the sparse signal respectively. $N_{S E}$ and $N_{S T}$ are the number of estimated support and the number of total support, respectively, both for the sparse signal. Similarly, $N_{O E}$ and $N_{O T}$ are the number of estimated sparsity order and the number of total sparsity order.

From Figure 1, we can see that the estimation performance of the sparsity order estimation is much higher than that of the support estimation and the reconstruction, while keeping the same simulation parameters. When $M=40, N=128, K=5, m=30$ and $\mathrm{SNR}=20 \mathrm{~dB}$, the correct rate of the sparsity order estimation is about $40 \%$ higher than that of the support estimation, and is about $180 \%$ higher than that of the reconstruction.

Furthermore, it can be seen in this simulation that the estimation performance based on the sparsity order can keep to be good performance even when the number of measurements is very low. Such estimation indicates that we can build the detection using the sparsity order with fewer number of measurements.

The sparsity order has been used to optimize the signal processing in [11] and signal reconstruction in [10]. In [11], the sparsity order is used to determine whether the signal is sparse or not, and different signal processing approach is selected based on the result. In [10], the number of compressive measurements needed for reconstruction is decided according to the estimation of the sparsity order. In this paper, we use it to detect the presence or absence of a sparse signal. Compared with support detection, this method only solves the simple detection problem, not the classification problem, but it needs fewer 
measurements than support detection.

\subsection{Sparsity Order Detection Algorithm}

The sparsity order detection (SOD) used greedy algorithms to get the larger coefficients and compute the sparsity order. Different from the reconstruction method and the support detection, the SOD only calculates the number of the coefficients, that is, the sparsity order of the target signal. If the number of the sparsity order is greater than the given threshold, the SOD indicates that the sparse signal is present. Otherwise, such sparse signal is absence. The SOD method is simple because it needs fewer measurements to solve the detection problem. The algorithm of SOD is provided as follows,

1. Initialize the residual $\mathbf{r}_{0}=\mathbf{y}$, the measurement matrix is $\mathbf{V}$. Initialize the iteration counter $t=1$ and the sparsity order $S_{s o}=0 . \mathbf{V}_{t}, \theta_{t}$ is the set of selected vectors of the measurement matrix or the approximation coefficients.

2. Select the measurement vector that maximizes the value of the projection of the residual onto $\mathbf{V}$,

$$
\begin{gathered}
\mathbf{v}_{t}=\arg \max _{i=1, \ldots \ldots, Z} \frac{<\mathbf{r}_{t-1}, \mathbf{v}_{i}>}{\left\|\mathbf{v}_{i}\right\|} . \\
\mathbf{V}_{t}=\left[\mathbf{V}_{t-1}, \mathbf{v}_{t}\right] .
\end{gathered}
$$

3. Update the residual and the estimate of the coefficient for the selected vector

$$
\begin{aligned}
\theta(t) & =\frac{\left\langle\mathbf{r}_{t-1}, \mathbf{v}_{t}>\right.}{\left\|\mathbf{v}_{t}\right\|^{2}} . \\
\theta_{t} & =\left[\theta_{t-1}, \theta(t)\right] . \\
\mathbf{r}_{t} & =\mathbf{r}_{0}-\theta_{t} \mathbf{V}_{t} .
\end{aligned}
$$

4. If $\theta(t)>\gamma$ then $S_{s o}=S_{s o}+1$ where $\gamma=(\mu+\delta) / 2, \gamma$ is the threshold of the sparsity order, $\mu$ is the average absolute value of the signal and $\delta$ is the standard deviation of the noise.

5. Increase $t$, If $t<T, T$ is the iteration times, then go to Step 2; otherwise end the algorithm.

6. If $1 \leq S_{\text {so }} \leq K$, the signal is presence, or it is absence. The $K$ is the maximum value of the sparse signal.

\section{Simulations}

This section presents simulation results to verify the detection performance and sampling cost of the proposed SOD, using support detection as a benchmark.

Consider a signal $\mathrm{x} \in \mathbb{R}^{N}, N=128$, which is K-sparse in the representation basis $\boldsymbol{\Psi}, K=$ 5 and such sparse signal is mixed with a Gaussian noise. In order to discuss easily and without loss of generality, we can use the identity matrix as the representation basis, i.e., $\boldsymbol{\Psi}=\mathbf{I}^{N \times N}$. We acquire the compressive measurements $\mathbf{y} \in \mathbb{R}^{M}$ from the random matrix $\boldsymbol{\Phi} \in \mathbb{R}^{M \times N}, M<N$, the entries of the random matrix are independent and identically distributed according to Gaussian probability distributions. The compressive measurements 


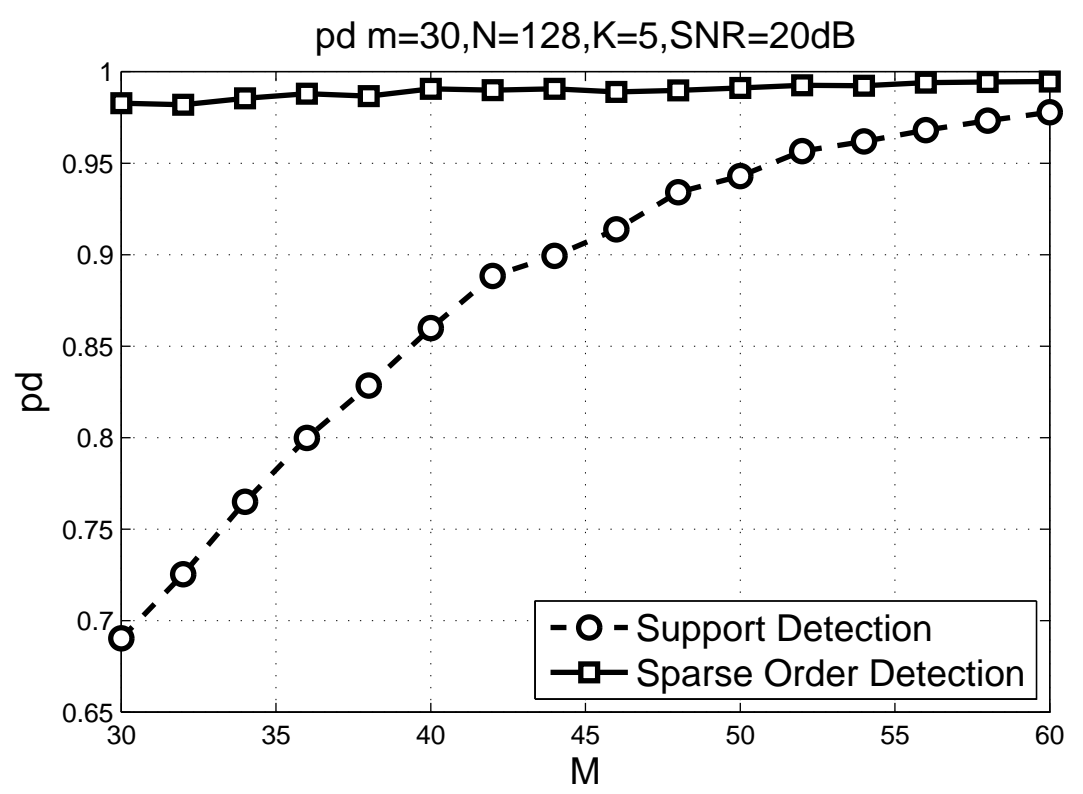

Figure 2. Detection Performance of SOD

have been shown in Eq. 9. The sparsity order detection and the support detection are all based on greedy algorithms, $m$ is the iterative times in these greedy algorithms.

Figure 2 indicates the probability detection of different methods with the number of measurements. We use greedy algorithms to estimate the sparsity order and the support. From Figure 2, we can find that the proposed sparsity order detection has higher probability detection than the support detection with the same number of measurements. With the decrease of the number of measurements, the detection performance of the support detection is becoming worse, but this sparsity order detection still can maintain better performance. Because the sparsity order detection only needs to identify the number of nonzero elements of the sparse signal, not the position or amplitude of each nonzero element, it needs fewer measurements than the support detection.

Figure 3 indicates the false alarm probability of different methods with the number of measurements. From Figure 3 we can see that the false alarm probability of the sparsity order detection is lower than the support detection. Under the conditions of fewer measurements, the false alarm probability of the sparsity order detection still remains at a low level. When the sparse signal is absence, the noise uniformly distributes in every vector of the measurement matrix. So the sparsity order of the measurements is zero.

\section{Conclusion}

A new sparse signal detection scheme based on the sparsity order has been discussed in this paper. The proposed scheme propose a new detection method based on the sparsity order. This is the first time to used the sparsity order to solve the sparse signal detection. The sparsity order detection solves the detection problem directly from the compressive measurements, does not need to reconstruct the signal beforehand. Because it only needs to identify the number of nonzero elements of the sparse signal, not the position or amplitude of each nonzero element, it needs fewer measurements than reconstruction or the 


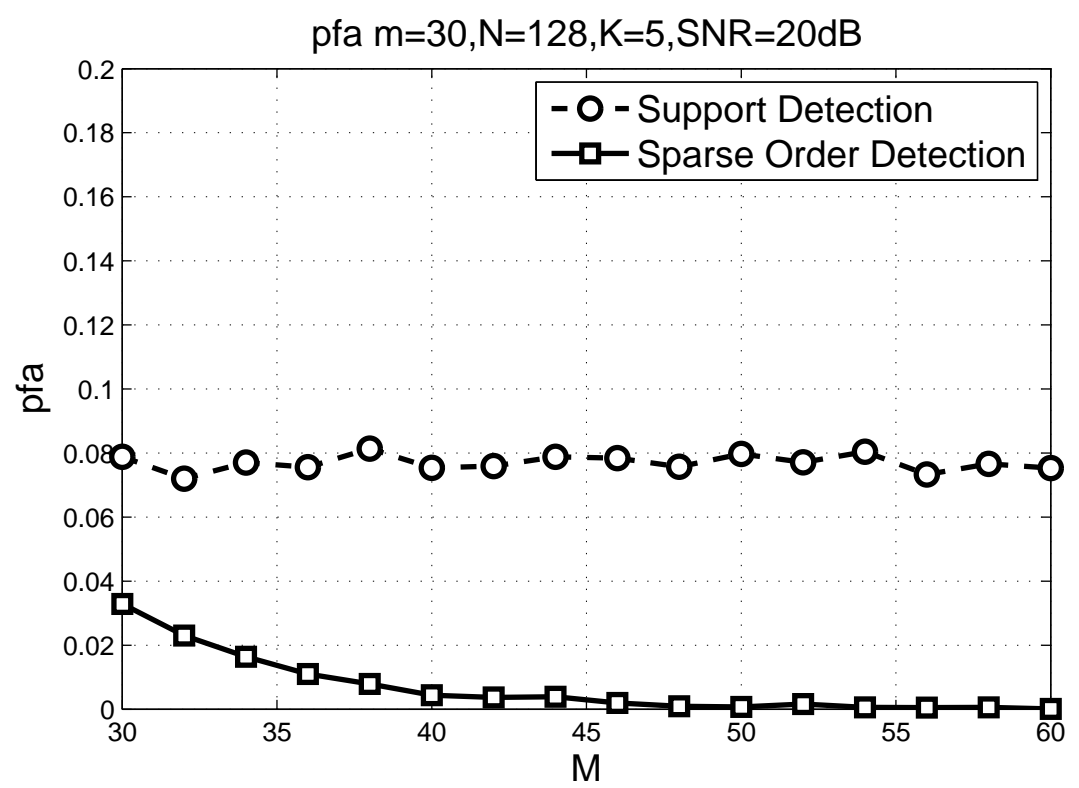

Figure 3. The false alarm probability with the number of measurements

support detection. The simulation results show the SOD has good performance than support detection with fewer measurements.

\section{References}

[1] D. Donoho, "Compressed sensing". IEEE Transactions on Information Theory, vol.42,no.4,(2006), pp.1289-1306.

[2] E. Candes and M. Wakin, "An Introduction To Compressive Sampling", IEEE Signal Processing Magazine, vol.25,no.2,(2008), pp.21-30.

[3] Jia (Jasmine) Meng, Wotao Yin, Husheng Li, Ekram Hossain and Zhu Han, "Collaborative Spectrum Sensing from Sparse Observations in Cognitive Radio Networks", IEEE Journal On Selected Areas In Communications vol.29,no.2,(2011), pp.327-337.

[4] Jia Meng, Husheng Li and Zhu Han, "Sparse Event Detection in Wireless Sensor Networks using Compressive Sensing", Proceedings of the 43rd Annual Conference on Information Sciences and Systems, Baltimore, MD, (2009), pp.181-185.

[5] Haifeng Zheng, Shilin Xiao and Xinbing Wang, "Sequential Compressive Target Detection in Wireless Sensor Networks", Proceedings of IEEE International Conference on Communications, Kyoto,(2011), pp.1-5.

[6] Mark A. Davenport, Michael B. Wakin and Richard G. Baraniuk, "Detection and Estimation with Compressive Measurements", Rice University ECE Technical Report TREE 0610, (2006), pp.1-16.

[7] Mark A. Davenport, Petros T. Boufounos, Michael B. Wakin and Richard G. Baraniuk, "Signal Processing With Compressive Measurements", IEEE Journal of Selected Topics in Signal Processing vol.4,no.2,(2010) pp.445-460. 
[8] Marco F. Duarte, Mark A. Davenport, Michael B. Wakin and Richard G. Baraniuk, "Sparse signal detection from incoherent projections", Proceedings of IEEE International Conference on Acoustics, Speech, and Signal Processing, Toulouse, France, (2006), pp.305-308.

[9] Alyson K. Fletcher, Sundeep Rangan and Vivek K Goyal, "Ranked Sparse Signal Support Detection", IEEE Transactions On Signal Processing, vol.60,no.11,(2012), pp.59195931.

[10] Yue Wang, Zhi Tian and Chunyan Feng, "Sparsity Order Estimation and its Application in Compressive Spectrum Sensing for Cognitive Radios", IEEE Transactions on Wireless Communications, vol.11,no.6,(2012), pp.2116-2124.

[11] Byonghyo Shim and Byungkwen Song, "Multiuser Detection via Compressive Sensing" IEEE Communications Letters, vol.16,no.7,(2012), pp.972-974.

[12] E. Cands and T. Tao, "Decoding by linear programming", IEEE Transactions on Information Theory, vol.51,no.12,(2005), pp. 4203-4215.

[13] S. Chen, D. Donoho and M. Saunders, "Atomic decomposition by basis pursuit. SIAM Journal on Scientific Computing", vol.20,no.1,(1998), pp.33-61.

[14] S. Mallat and Z. Zhang, "Matching pursuits with time-frequency dictionaries. IEEE Transactions on Signal Processing", vol.41,no.12,(1993), pp.3397-3415.

[15] Joel A. Tropp and Anna C. Gilbert, "Signal Recovery From Random Measurements Via Orthogonal Matching Pursuit", IEEE Transactions on Information Theory, vol.53,no.12,(2007), pp.4655-4666.

[16] D. Needell and J. Tropp, "CoSaMP: Iterative signal recovery from incomplete and inaccurate samples", Applied and Computational Harmonic Analysis,vol.26,no.3,(2009), pp.301-321.

[17] Yi Chen, Nasser M. Nasrabadi and Trac D. Tran, "Sparse Representation for Target Detection in Hyperspectral Imagery", IEEE Journal Of Selected Topics In Signal Processing, vol.5,no.3,(2011), pp.629-639.

[18] A. K. Fletcher and S. Rangan, "Orthogonal matching pursuit: A Brownian motion analysis", IEEE Transactions on Signal Process, vol.60,no.3,(2012), pp.1010-1021.

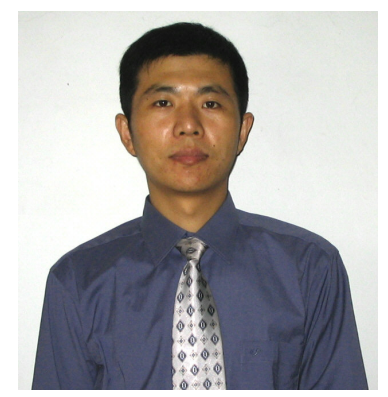

Author

Shaohua Qin is a Ph.D. student in School of Control Science and Engineering, Shandong University, China. He is also a lecturer in College of Physics and Electronics, Shandong Normal University. His research interests are Compressed Sensing and Wireless Sensor Networks. email:qsh@sdu.edu.cn. 